\title{
Reverse Marginal Mandibulectomy for Submandibular Gland Tumor
}

\author{
Garima Daga ${ }^{1}$, Rajib Paul ${ }^{2}$, Ghanashyam Mandal ${ }^{3}$, Rajeev Kumar ${ }^{4}$
}

\begin{abstract}
Segmental resection of mandible was a preferred approach in oral cancers with proximity to the bone, for adequate resection margins and fear of local recurrence as it was thought to spread via the periosteal lymphatics. However, with a better understanding of oral cancer spread, mandibular preserving strategies are being practiced in which only a portion of the mandible is excised with preservation of bony continuity. This improves overall functional and cosmetic outcomes. Conservative mandibular resections including the classical rim, reverse, sagittal, and oblique types have been described in contemporary literature. We report reverse marginal mandibulectomy in a case of submandibular gland tumor and review the clinical and pathological basis of the selection of cases suitable for mandibular preservation strategy.

Keywords: Conservative mandibular resection, Reverse marginal mandibulectomy, Segmental resection, Submandibular gland.

International Journal of Head and Neck Surgery (2021): 10.5005/jp-journals-10001-1426
\end{abstract}

\section{INTRODUCTION}

In addition to facial cosmesis, mandible plays an important role in speech and mastication. Mandibular resection for any indication leads to significant morbidity-both in respect of chewing and speech. With a better understanding of the pathology of oral cancers, the original mandibular resections, i.e., hemimandibulectomy or full-thickness segmental mandibulectomy have been modified. Conservative mandibular resections with preservation of part of the bone are being performed. This provides much better functional and cosmetic outcomes and avoids major microvascular reconstructive procedures. Various techniques of conservative mandibular resections including classic, sagittal, oblique, and reverse have been described but there is a paucity of literature regarding the same. We hereby report reverse marginal mandibulectomy, in a case of squamous cell carcinoma of the left submandibular salivary gland.

\section{Materials and Methods}

A 69-year-old non-edentulous woman presented with gradually increasing painless swelling in the left submandibular gland region. She underwent an incisional biopsy which revealed a mucoepidermoid carcinoma of the submandibular gland. Magnetic resonance imaging was done for local staging and revealed an altered signal intensity lesion of $4.4 \times 3.3 \times 3.0 \mathrm{~cm}$ in the left submandibular gland extending anteriorly to left sublingual space and floor of mouth with loss of fat planes with left hyoglossus, mylohyoid and digastrics muscles, and ipsilateral genioglossus muscle. Superiorly lesion was closely abutting inferomedial cortex of mandibular body. The left facial artery was encased within the lesion with extension to the subcutaneous plane. There was no regional lymphadenopathy (Fig. 1).

\section{Results}

She underwent wide local excision of left submandibular gland with reverse marginal mandibulectomy with left modified neck lymph node dissection (Figs 2 and 3). Left sternocleidomastoid
1,2,4 Department of Surgical Oncology, Rajiv Gandhi Cancer Institute and Research Centre, New Delhi, India

${ }^{3}$ Department of Head and Neck Oncology, Rajiv Gandhi Cancer Institute and Research Centre, New Delhi, India

Corresponding Author: Garima Daga, Department of Surgical Oncology, Rajiv Gandhi Cancer Institute and Research Centre, New Delhi, India, Phone: +91 9930484364, e-mail: narsinghbaba@yahoo. co.in

How to cite this article: Daga G, Paul R, Mandal G, et al. Reverse Marginal Mandibulectomy for Submandibular Gland Tumor. Int J Head Neck Surg 2021;12(2):86-88.

Source of support: Department of Surgical Oncology, Rajiv Gandhi Cancer Institute and Research Centre, New Delhi, India

\section{Conflict of interest: None}

muscle flap was used as a buttress floor of the mouth. The muscle was sutured deep to the mandible after creating deep pockets with a drill bit (Fig. 4). The perioperative course was uneventful.

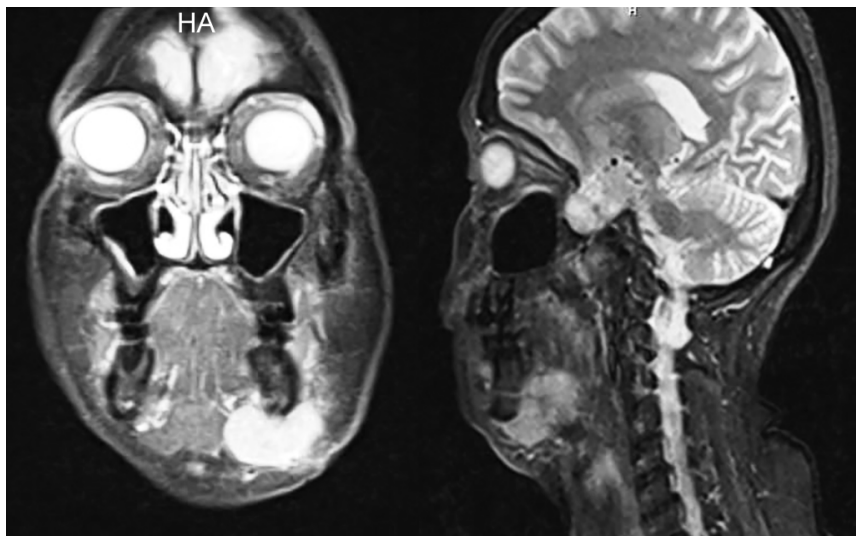

Fig. 1: Magnetic resonance imaging: T2 image with altered signal intensity lesion of $4.4 \times 3.3 \times 3.0 \mathrm{~cm}$ in left submandibular gland extending anteriorly to left sublingual space and floor of the mouth. Superiorly lesion is closely abutting inferomedial cortex of mandibular body

(0) The Author(s). 2021 Open Access This article is distributed under the terms of the Creative Commons Attribution 4.0 International License (https:// creativecommons.org/licenses/by-nc/4.0/), which permits unrestricted use, distribution, and non-commercial reproduction in any medium, provided you give appropriate credit to the original author(s) and the source, provide a link to the Creative Commons license, and indicate if changes were made. The Creative Commons Public Domain Dedication waiver (http://creativecommons.org/publicdomain/zero/1.0/) applies to the data made available in this article, unless otherwise stated. 


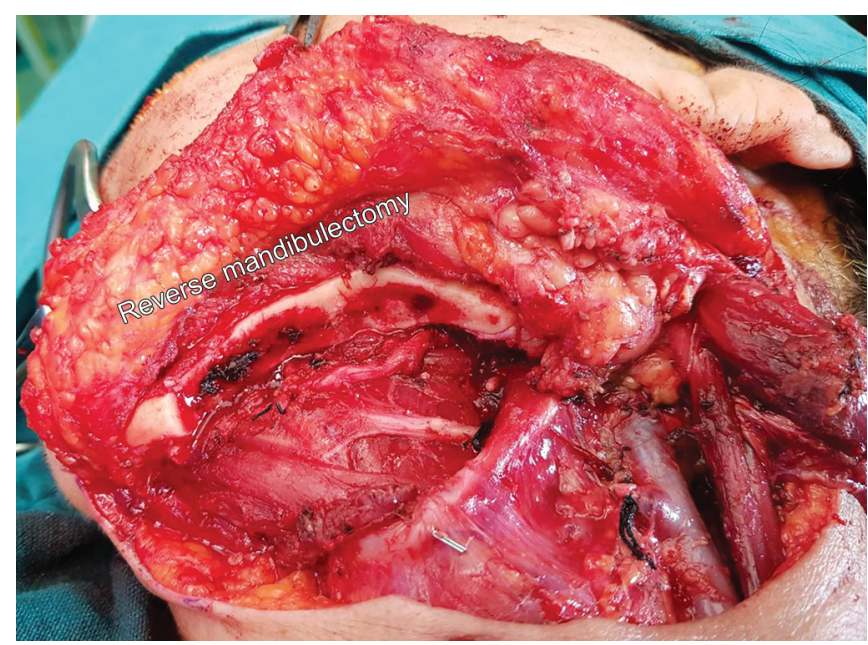

Fig. 2: Intraoperative photograph demonstrating reverse marginal mandibulectomy. The bony contour of the mandible is maintained

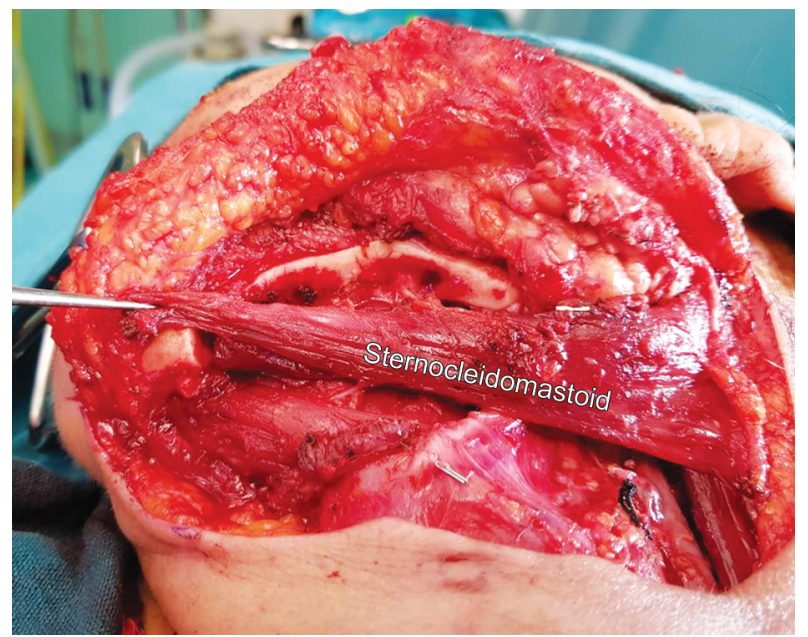

Fig. 4: Buttressing of the floor of mouth with left sternocleidomastoid muscle flap

Histopathology revealed keratinizing (Fig. 5) type squamous cell carcinoma of submandibular gland with clear margins, pT2N1. The patient is doing fine till 3 months of follow-up.

\section{Discussion}

The mandible is one of the strongest bones and plays an important role in mastication, speech, and facial cosmesis. Management of salivary gland cancers that involve the mandible needs a specific understanding of the actual pattern of spread. With a better understanding of the spread of these cancers, the original mandibular resection, i.e., hemimandibulectomy or full-thickness segmental mandibulectomy was amended to preserve part of the bone. This aided the employment of mandibular sparing approaches like marginal mandibulectomy and mandibulotomy, against segmental or hemimandibulectomy with much better functional and cosmetic outcomes. 'The traditional approaches led to major functional problems, as the mandibular continuity is lost. With the advent of mandibular sparing approaches, the major microvascular reconstruction with its associated morbidity is avoided.

A detailed preoperative clinical and radiological workup along with an understanding of the pattern of spread and routes

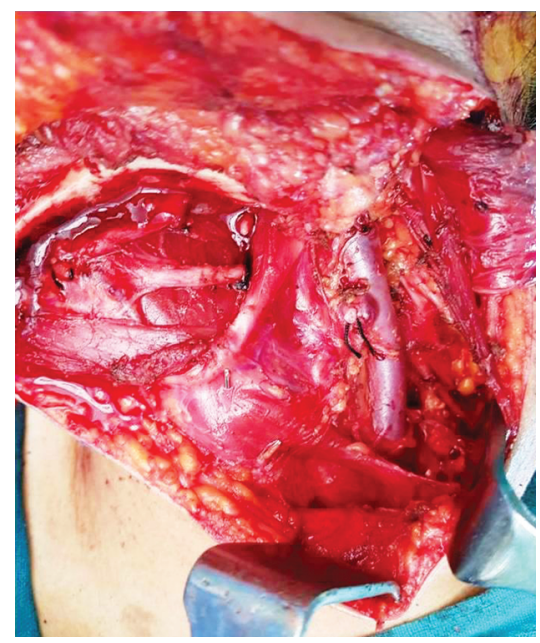

Fig. 3: Left modified neck lymph node dissection

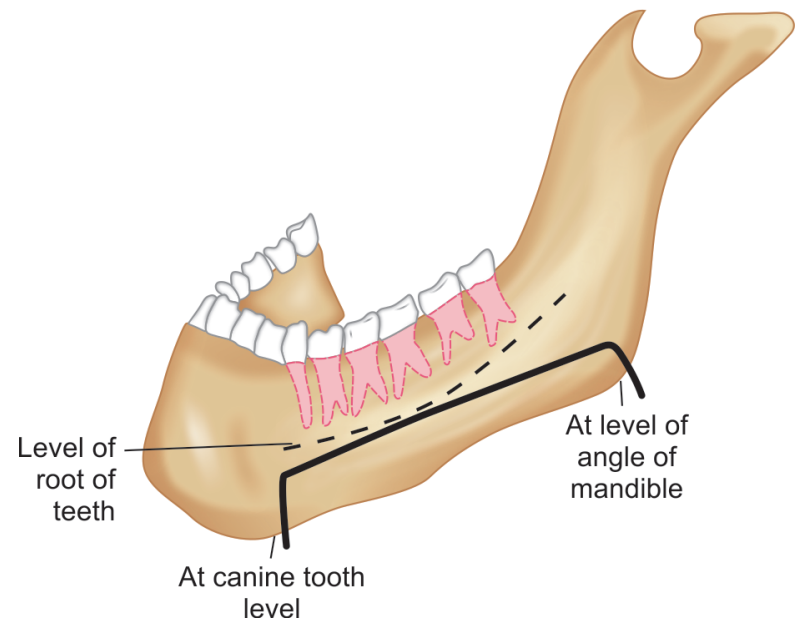

Fig. 5: Line diagram depicting left-sided reverse mandibulectomy

of invasion is paramount in deciding the appropriate level and extent of mandibular resection in submandibular gland tumors. Literature supports comparable local control rates between marginal and segmental mandibulectomy and hence in carefully selected patients, marginal mandibulectomy is an oncologically safe procedure. It provides good local control and provides a better quality of life. ${ }^{2}$ Guerra et al. retrospectively analyzed data of 106 cases and concluded that the resection margin, the involvement of bone, and the size of mandibulectomy affected the prognosis for oral carcinomas. Mandibular conservation is oncologically safe in early stage head and neck cancers. ${ }^{3}$

Selection of cases for mandibular preservation techniques is paramount without a compromise on oncologic outcomes. O'Brien et al. in their series of 127 patients performed marginal mandibulectomy in 97 patients and concluded that involved soft tissue margins are predictive of poor local control or decreased survival rates among patients with oral and oropharyngeal cancers. As long as marginal mandibulectomy does not lead to compromise of soft tissue margins conservative resection of the mandible is safe. Segmental resection should be reserved for patients with extensive bone invasion or those with limited invasion in a thin atrophic mandible. ${ }^{4}$ 
Various types of conservative mandibular resections have been described in the literature as classical (Rim), sagittal, reverse, and oblique marginal mandibulectomy. ${ }^{1}$ Various criteria have been proposed in the literature for selecting marginal mandibulectomy. Randall et al. recommended that it could be done with no radiological evidence of bone erosion and $<50 \%$ of the mandible's circumference was involved by tumor. ${ }^{5}$ The rim resection of the upper border of the mandible including the alveolar canal was proposed by McGregor and MacDonald as an alternative to full segmental resection. ${ }^{6}$ For lesions that were anatomically closer to the lower border of the mandible, such as submental nodes eroding into its periosteum, the same principle was used to resect the lower rim while preserving continuity in the upper border. This resection was termed the reverse marginal mandibulectomy and the same was done in the present case. There is always a risk of exposing the dental roots and hence meticulous resection is paramount without compromising the oncological outcomes. ${ }^{1}$ In the present case, reverse marginal mandibulectomy was done with the preservation of the dental roots.

Sometimes the sagittal resection is done in which either the inner or the outer cortex of the mandible is excised. This is preferred in tumors of the gingivobuccal or gingivolabial (lateral) sulcus when the mandible is not involved but is in proximity to the tumor. Oblique marginal mandibulectomy is done in tumors of the floor of the mouth abutting the mandible. Shaha used this in his series of 65 patients with carcinoma floor of the mouth, of which 22 underwent marginal mandibulectomy. Most of these were oblique types including the upper rim and inner cortex of the mandible. ${ }^{7}$

Mandibular preservation strategies have led to improved functional outcomes with preservation of oncological outcomes.
Preoperative staging including clinical, radiological workup and hence case selection is imperative in conservative mandibular resections.

\section{ACKnowledgment}

We would like to thank Rajiv Gandhi Cancer Institute and Research Center, New Delhi, India.

\section{References}

1. Ghosh SK, Ghosh SK. Conservative mandibular resections for oral cavity malignancies: a review. Nepal Med Coll J. 2010;12(2): 119-122.

2. Rao LP, Shukla M, Sharma V, et al. Mandibular conservation in oral cancer. Surg Oncol 2012;21(2):109-118. DOI: 10.1016/j. suronc.2011.06.003.

3. Guerra MF, Gías LN, Campo FR, et al. Marginal and segmental mandibulectomy in patients with oral cancer: a statistical analysis of 106 cases. J Oral Maxillofac Surg 2003;61(11):1289-1296. DOI: 10.1016/ S0278-2391(03)00730-4.

4. O'Brien CJ, Adams JR, McNeil EB, et al. Influence of bone invasion and extent of mandibular resection on local control of cancers of the oral cavity and oropharynx. Int J Oral Maxillofac Surg 2003;32(5):492-497. DOI: 10.1016/S0901-5027(03)90420-6.

5. Randall CJ, Eyre J, Davies D, et al. Marginal mandibulectomy for malignant disease: indications, rationale and results. J Laryngol Otol 1987;101:676-684.

6. McGregor AD, MacDonald DG. Routes of entry of squamous cell carcinoma to the mandible. Head Neck Surg 1988;10(6):29-31. DOI: 10.1002/hed.2890100604

7. Shaha AR. Marginal mandibulectomy for carcinoma of the floor of the mouth. J Surg Oncol 1992;49(2):116-119. DOI: 10.1002/jso.2930490211. 